\title{
Rezension: Monika Simmler, Normstabilisierung und Schuldvorwurf
}

\section{Gian Ege *}

Über das Wesen der strafrechtlichen Schuld wird seit jeher gestritten. Auf den ersten Blick scheint immerhin Einigkeit darüber zu bestehen, dass Schuld die Hauptvoraussetzung für eine Strafe ist (nulla poena sine culpa). Allerdings gilt dies nur bedingt und das Schuldprinzip ist verschiedenen Angriffen ausgesetzt. In ihrer Dissertation verortet Monika Simmler die Funktion des Strafrechts in der Normstabilisierung und zeigt auf, dass dem Schuldvorwurf in diesem Zusammenhang entscheidende Bedeutung zukommt.

Zitiervorschlag: Gian Ege, Rezension: Monika Simmler, Normstabilisierung und Schuldvorwurf, in: sui-generis 2018, S. 327

URL: $\quad$ sui-generis.ch/77

DOI: $\quad$ https://doi.org/10.21257/sg.77

* Dr. iur. Gian Ege (gian.ege[at]rwi.uzh.ch), Oberassistent für Strafrecht und Strafprozessrecht am Rechtswissenschaftlichen Institut der Universität Zürich. 
Die Schuld ist wohl das zentrale Element unseres Strafrechtssystems. Sie ist Voraussetzung für die Bestrafung eines Delinquenten (Strafbegründungsschuld, Art. 19 Abs. 1 StGB) und bildet die Grundlage für die Höhe der Strafe (Strafzumessungsschuld, Art. 47 Abs. 1 StGB). Allerdings verliert das Schuldprinzip seit Längerem seine Unantastbarkeit - wenn es diese denn jemals besessen hat - und kommt zunehmend in Bedrängnis. Einerseits bestehen bereits verschiedene Bestimmungen, welche das Schuldprinzip einschränken, und andererseits wird auf politischer Ebene auf dessen weitere Aushöhlung hingearbeitet. ${ }^{1}$ Für Ersteres sind beispielsweise die Regelung über die Fahrzeughalterhaftung² sowie das Massnahmenrecht3 zu nennen.

Vor diesem Hintergrund ist es durchwegs angemessen und zeitgemäss, dass sich Monika Simmler in ihrer von Martin Killias betreuten Zürcher Dissertation vertieft der Bedeutung der Schuld widmet und untersucht, inwiefern sie verzichtbar oder austauschbar ist. Der Arbeit liegt die These zu Grunde, «dass das traditionelle Schulderfordernis durch alternative Legitimationsmodelle ersetzt werden kann, solange das Strafrechtssystem sei-

\footnotetext{
So reichte Andrea Martina Geissbühler am 23. September 2015 eine Motion (15.3932) ein, wonach die Strafmilderung für unter Alkohol- beziehungsweise Drogeneinfluss stehende Täter abgeschafft werden sollte. Die Motion wurde vom Nationalrat am 1. Juni 2017 mit 121 zu 65 Stimmen abgelehnt. Die gleiche Nationalrätin hatte bereits am 2. Dezember 2012 eine parlamentarische Initiative (09.500) zur Streichung von Artikel 19 und 20 StGB eingereicht, der jedoch keine Folge gegeben wurde.

Art. 6 OBG.

3 So können Massnahmen explizit auch gegen schuldunfähige Straftäter verhängt werden, Art. 19 Abs. 3 StGB.
}

ne Funktion in der Gesellschaft nach wie vor adäquat erfüllt» (S. 4).

3 Im ersten Teil ihrer Arbeit beleuchtet die Autorin das Schuldprinzip und dessen Variabilität sowie die angelsächsische Rechtsfigur der strict liability als Angriff darauf (S. 9 ff.). Als Grundlage für die weitere Untersuchung beschreibt sie das Verständnis des Schuldprinzips in den deutschsprachigen und den angelsächsischen Rechtsordnungen. Hier zeigt sich eine zentrale Schwierigkeit des Vorhabens. So unterscheiden sich die Schuldkonzeptionen zwischen diesen Rechtsordnungen und auch innerhalb derselben hat sich kein durchwegs konsensuales Verständnis etabliert. Anschliessend wendet sich Simmler den strict liability offences als "Ausnahme von der Regel» (S. 53) zu. Die Autorin erläutert insbesondere die theoretische Rechtfertigung dieser Deliktformen sowie die dagegen erhobene Kritik und zeigt Anwendungsbeispiele auf. Um dem unterschiedlichen Schuldverständnis zu begegnen und sich dem Untersuchungsgegenstand weiter anzunähern, unternimmt Simmler eine systemtheoretische Analyse der Schuld. Damit will sie aufzeigen, «dass trotz pluralistischen Konzeptionen eine systemfunktionale Einheitlichkeit besteht» (S. 115).

4 Im zweiten Teil wird das Strafrecht als soziales System betrachtet (S. 133 ff.). Dafür legt die Autorin zunächst die systemtheoretischen Grundlagen dar und beschreibt die gesamte Rechtsordnung als soziales System. Die allgemeinen Ausführungen zur Systemtheorie sind für den strafrechtlichen Leser nicht leicht $\mathrm{zu}$ ergründen. Es erscheint zuweilen frag- 
lich, inwiefern sie für den weiteren Gang der Untersuchung notwendig sind, und man wünscht sich eine konzisere Darstellung. Eingängiger ist das Kapitel über das Strafrechtssystem und die Funktion der Strafe. Die Autorin legt die Operationsweise des Strafrechtssystems (schuldig / nicht schuldig) sowie dessen selbstreferentielle Geschlossenheit dar und versteht das Strafrecht somit als eigenständiges soziales Subsystem. Davon grenzt sie das Massnahmenrecht ab, da dieses eine andere Funktion erfülle und «ein soziales System nicht gleichzeitig zwei Funktionen erfüllen kann» (S. 185). In der Folge macht sich Simmler auf die Suche nach der Funktion der Strafe, welche sie in der Normstabilisierung und damit in der positiven Generalprävention verortet. Damit werde, so die Autorin, keine zukünftige Verhaltenssteuerung, sondern eine Vergangenheitsbewältigung (Ausgleich einer nicht erfüllten Erwartung) bezweckt. Somit gehe es bei einer entsprechend positiv generalpräventiven Ausrichtung des Strafrechts nicht um Verbrechensverhütung, sondern um eine «Reaktion auf das Verbrechen» (S. 205).

5 Der dritte Teil der Untersuchung widmet sich der Funktion des Schuldvorwurfs im Rahmen der Normstabilisierung (S. $221 \mathrm{ff}$.). Simmler legt dar, dass einzig eine schuldangemessene Strafe normstabilisierend wirken kann, da nur ein schuldhafter Normbruch die Normstabilität beeinträchtigt. Damit begründet die Schuld die Notwendigkeit der Strafe (Strafbegründungsschuld). Gleichzeitig soll die Strafe nicht über das Mass der Normdestabilisierung hinausgehen, womit ein Proportionalitätsprinzip festgelegt wird (Strafzumessungsschuld). Als dritte Komponente beinhaltet das
Schuldprinzip auch eine verfahrensrechtliche Komponente. So muss die Notwendigkeit der Strafe - und damit die Schuld des Täters - in einem tauglichen Verfahren festgestellt werden. Sodann werden die verschiedenen Elemente des Schuldvorwurfs dargestellt. Den vorangehenden Überlegungen folgend ist Schuld die $\mathrm{Zu}-$ schreibung der Verantwortung für eine Normdestabilisierung. Ein Schuldvorwurf wird demnach «platziert, wenn eine Norm destabilisiert wurde. Eine Norm wurde nur dann destabilisiert, wenn erstens ein taugliches Subjekt (Personalität) vorliegt, dem zweitens ausreichend situative Kompetenz zugeschrieben wird, welche die Vermeidung des Fehlers erlaubt hätte (Kompetenz) und wenn die Tat darüber hinaus drittens eine Infragestellung der Norm kommuniziert (Mitteilung)» (S. 255). Bei der Personalität geht es vorweg darum, ob eine Person überhaupt Adressat normativer Erwartungen sein kann (Schuldfähigkeit). Die notwendige Kompetenz liegt vor, wenn der Täter anders hätte handeln können. Schliesslich muss die Tat die Infragestellung der Norm mitteilen. Dies hänge mehrheitlich vom subjektiven Tatbestand ab: Wer vorsätzlich handle, teile damit eine gravierendere Normdestabilisierung mit. Dabei bleiben die Ausführungen teilweise etwas unklar. Die Autorin lässt offen, ab wann von einer für den Schuldvorwurf notwendigen Mitteilung ausgegangen werden kann. Zudem berücksichtigt sie die dogmatische Stellung des subjektiven Tatbestandes zu wenig. Kann einem Täter nämlich kein subjektiver Tatbestand nachgewiesen werden, fehlt bereits das Unrecht der Tat, weshalb sich die Schuldfrage nicht stellt. Demzufolge betrifft die Unterscheidung von Vorsatz und Fahrlässigkeit im Rahmen des 
Schuldvorwurfs vorwiegend das Strafzumessungsverschulden. 4

Im vierten Teil werden die Normstabilisierung und die Variationen der strikten Verantwortlichkeit untersucht (S. $311 \mathrm{ff}$.). Um das Verhältnis der strict liability zum Schuldprinzip zu klären, kategorisiert die Autorin zunächst die verschiedenen Variationen der strict liability. In Ermangelung überzeugender Alternativen erarbeitet sie eine eigene Differenzierung, die sich grösstenteils an den bereits dargelegten Elementen der Schuld orientiert. Diese Unterarten der strict liability werden untersucht und mit Beispielen belegt. Daneben wird aufgezeigt, dass auch in den deutschsprachigen Rechtsordnungen Ansätze bestehen, die zumindest teilweise mit strict liability offences vergleichbar sind - dazu zählen erfolgsqualifizierte Delikte, objektive Strafbarkeitsbedingungen, Rauschtatbestände, die Versuchsstrafbarkeit sowie die Unternehmensstrafbarkeit. Immer wieder kommt Simmler zum Schluss, dass die einzelnen Formen der strict liability nicht im Einklang mit dem Schuldprinzip stehen und somit dysfunktional sind. So kommt die Autorin nicht überraschend zum Schluss, dass die strict liability offences «nicht mit der idealen Funktions-

\footnotetext{
Dies zeigt sich in den unterschiedlichen Strafrahmen zwischen Vorsatz- und Fahrlässigkeitsdelikten, vgl. Marcel A. Niggli/Stefan Maeder, in: Niggli/Wiprächtiger (Hrsg.), Basler Kommentar Strafrecht I, 3. Aufl., Basel 2013, Art. 12 N 16 ff. Wobei man sich fragen kann, ob diese Differenzierung nicht auf einem unterschiedlichen Unrechtsgehalt statt einem unterschiedlichen Schuldvorwurf beruht, vgl. dazu z.B. Günter Stratenwerth, Schweizerisches Strafrecht, Allgemeiner Teil I, 4. Aufl., Bern 2011, § 9 N 58 ff. Unzweifelhaft wird das Strafzumessungsverschulden vom jeweiligen Grad des Vorsatzes bzw. der Fahrlässigkeit beeinflusst, Daniel Jositsch/Gian Ege/Christian Schwarzenegger, Strafrecht II, 9. Aufl., Zürich 2018, 111.
}

weise des Strafrechtssystems in Einklang zu bringen sind» (S. 402).

7 Im abschliessenden fünften Teil wird ein funktionales Schuldprinzip als $\mathrm{Ga}$ rant der Systemstabilität dargestellt (S. 417 ff.). Die Autorin bekräftigt die Stabilisierung normativer Erwartungen als Funktion des Strafrechts und den Schuldvorwurf als entsprechende Operationsweise. Durch die Schuld sei zu entscheiden, «ob für die Normstabilisierung Strafe notwendig ist und in welchem Ausmass sie erforderlich ist» (S. 421). In der Folge wird ein funktionaler Schuldbegriff aufgearbeitet. Dabei kommt Simmler aufgrund ihrer Untersuchung zum Schluss, dass eine Funktionalisierung des Schuldvorwurfs nicht systeminhärent sei, sondern durch Rechtsfiguren wie der strict liability gestört werden könne. Da jedoch nur eine Verantwortungszuschreibung die Stabilität und die Aufgabenerfüllung des Strafrechts gewährleiste, sei es aus «funktionaler Perspektive sinnvoll, am Schuldprinzip festzuhalten» (S. 452).

8 Damit befindet man sich wieder am Anfang: Die Ausgangsthese der Arbeit muss als widerlegt gelten. Das Schuldprinzip ist das Herzstück unseres Strafrechtssystems und damit überlebensnotwendig. Auch wenn die Autorin eine terminologische Umbenennung befürwortet (S. 452 f.), wird damit kein alternatives Legitimationsmodell vorgelegt, sondern am Bestehenden festgehalten. Die Frage nach dem Stellenwert der Schuld im Strafrecht wird somit in Übereinstimmung mit der herrschenden Auffassung beantwortet. Dies spricht keinesfalls gegen die Untersuchung. Wie einleitend gezeigt, ist die Themenauswahl durchaus 
angemessen und der Leser bleibt nicht ohne Erkenntnisgewinn zurück. Sowohl die systemtheoretische Analyse des Strafrechts und der Schuld als auch die Besprechung der Angriffe auf das Schuldprinzip am Beispiel der strict liability ermöglichen eine neue Perspektive auf die Frage nach dem Stellenwert der strafrechtlichen Schuld.

9 Der zusätzliche Wert ihrer Untersuchung zeigt sich auch auf «Nebenschauplätzen». Im Rahmen der Ausführungen zum Andershandelnkönnen erteilt Simmler den vorwiegend von Seiten der Neurowissenschaften erhobenen Einwänden gegen die Willensfreiheit und damit auch gegen das Schuldprinzip eine Abfuhr. Willensfreiheit ist in ihrem Schuldverständnis eine normative Konstruktion, weshalb an einem Andershandelnkönnen festgehalten werden kann.5 Gerade die Stellung der Schuld und dessen Elemente im sozialen System werden als Argument für die normative Setzung der Handlungsfreiheit verwendet. Damit wird die strafrechtliche Antwort auf die dagegen erhobenen Einwände ein weiteres Mal gestärkt. ${ }^{6}$

Die Breite der Untersuchung erweist sich jedoch zuweilen als zweischneidiges Schwert. Auf der einen Seite ermöglicht ihr Blick in verschiedene Rechtsordnungen die Berücksichtigung verschiedener

$5 \overline{\text { Der Schuldvorwurf an sich ist eine normative }}$ Konstruktion. Dabei ist das Andershandelnkönnen eine bloss hypothetische Beurteilung; der Täter hat faktisch nicht anders gehandelt. Für die Beurteilung wird dabei auf eine «massgeschneiderte» Durchschnittsperson abgestellt. Somit liegt dem Schuldvorwurf kein vollständiger Indeterminismus, sondern bloss eine Freiheitsannahme zugrunde, vgl. Gian Ege, Das Strafrecht und die Hirnforschung, ZStrR 2017, 299, $312 \mathrm{f}$. m.w.H.

6 Vgl. für weitere Einwände gegen die Kritik am Schuldprinzip, Ege (Fn. 5), 306 ff. m.w.H. theoretischer Überlegungen sowie die Beschäftigung mit unterschiedlichen Angriffen auf das Schuldprinzip. Dies bedingt andererseits, dass den dogmatischen Eigenheiten der untersuchten Rechtsordnungen nicht immer gerecht werden kann. An verschiedenen Stellen gewisse wurden bereits erwähnt wünscht man sich eine detailliertere Auseinandersetzung mit spezifischen Fragestellungen. Gerade dort, wo es um die Funktion von Strafrecht und Strafe geht, bleibt teilweise unklar, ob sich die Ausführungen auf die theoretische Fundierung des Strafrechts als solches oder auf die Strafe beziehen. Dabei lassen sich die Überlegungen der Strafrechtstheorien, die sich mit der Begründung des Strafrechts befassen, nicht eins zu eins auf die Straftheorien, die Sinn und Zweck von Strafen behandeln, übertragen. Dasselbe gilt natürlich auch vice versa. Diesbezüglich wäre eine feinere Unterscheidung angebracht.

11 Die Ausklammerung des Massnahmenrechts ist auf den ersten Blick eine Enttäuschung. Gerade bei diesem Teil des Sanktionenrechts 7 wird immer wieder über dessen Verhältnis zum Schuldprinzip gestritten. Bereits die gesetzliche Regelung macht klar, dass Massnahmen auch gegen schuldunfähige Täter angeordnet werden können (Art. 19 Abs. 3 StGB). Zudem fehlt bei den Massnahmen die begrenzende Wirkung der Schuld, weshalb sie zeitlich weit über schuldangemessene Strafen hinausgehen können. ${ }^{8}$ Die Begründung der Ausklamme-

7 Sowohl in der Schweiz (Art. 56 ff.) als auch in Deutschland ( $§ 63 \mathrm{ff}$.) ist das Massnahmenrecht im Strafgesetzbuch geregelt.

8 Einziger Beschränkungsmassstab ist die Verhältnismässigkeit, vgl. Jositsch/Ege/Schwarzenegger (Fn. 4), $172 \mathrm{f}$. 
rung des Massnahmenrechts ist zwar folgerichtig, weshalb die Ausklammerung des Massnahmenrechts der Autorin nicht zum Vorwurf gemacht werden kann. So sind Massnahmen nicht auf die Normstabilisierung gerichtet, weshalb sie von der Autorin konsequenterweise nicht dem Strafrecht zugeordnet werden. Damit ist sie nicht alleine; immer wieder wird bezweifelt, ob es sich beim präventiv ausgerichteten Massnahmenrecht tatsächlich um Strafrecht handelt. 9 Trotzdem wird damit ein gewichtiger Teil der Diskussion über den Stellenwert und die Bedeutung der Schuld (leider) gänzlich unbeachtet belassen.

Insgesamt legt Simmler die grundsätzliche Bedeutung des Schuldprinzips eindrücklich dar und zeigt auf, wie gefährlich Angriffe darauf - wie durch die strict liability oder ähnliche Rechtsfiguren im deutschsprachigen Raum - für das gesamte Strafrecht sein können. Gerade in einer Zeit, in der das Strafrecht einer vermehrt punitiven Grundhaltung gegenübersteht, ${ }^{10}$ ist diese Erkenntnis grundlegend.

«Normstabilisierung und Schuldvorwurf:
Eine Analyse der Funktion und Variabili-
tät der strafrechtlichen Schuld am Bei-
spiel der Strict Liability», Monika Simm-
ler, Carl Grossmann Verlag, Berlin/Bern
2018, ISBN: 978-3-941159-18-1 (ge-
druckte Ausgabe, Hardcover), ISBN:
978-3-941159-20-4 (gedruckte Ausgabe,
Paperback), ISBN: 978-3-941159-19-8
(e-Book, Open Access)

«Normstabilisierung und Schuldvorwurf: Eine Analyse der Funktion und Variabilität der strafrechtlichen Schuld am Beispiel der Strict Liability», Monika Simmler, Carl Grossmann Verlag, Berlin/Bern 2018, ISBN: 978-3-941159-18-1 (gedruckte Ausgabe, Hardcover), ISBN: 978-3-941159-20-4 (gedruckte Ausgabe, (e-Book, Open Access)

9 Vgl. Marcel A. Niggli/Stefan Maeder, Die funktionale Stellung der Schuld in absoluten und relativen Straftheorien, in: Queloz/Niggli/Riedo (Hrsg.), Droit pénal et diversités culturelles, FS José Hurtado Pozo, Genf 2012, 297, 302 ff.

10 Vgl. Felix Bommer, Mass Incarceration in den Vereinigten Staaten von Amerika - ein Phänomen und seine Ursachen, ZStrR 2018, 117, 178; Martin Seelmann/Hans Wiprächtiger, Ausgekuschelt!?, AJP 2018, 734, 734 ff. 